\title{
Fast and adaptive detection of pulmonary nodules in thoracic CT images based on improved fuzzy c-means medical image segmentation and SVM classifier
}

\section{Ms.Sreesha Govind \#1, Mr. Rahul C \#2}

\#1 M.Tech Scholar, Department of CSE,LBS College of Engineering,Kasaragod,Kerala, \#2 Assistant Professor, Department of CSE,LBS College of Engineering,Kasaragod,Kerala

\section{ABSTRACT}

Lung cancer found to be the common cause of death among people all around the world. The chance of survival among people was increased with the early detection of lung cancer. There has been an increase in the survival rate from 14 to $40 \%$ when it is detected in its earlier stage. Computed Tomography (CT) can be more efficient than X-ray. To classify the present of lung cancer in an CT-images, a lung cancer detection system using image processing is used. In this method, MATLAB is used as the software tool for processing images.In image processing procedures, process such as image pre-processing, segmentation with procedure called as fuzzy cmeans(FCM) clustering and feature extraction with the help of GLCM matrix and classification by using ANN classifier have been performed. It is intended to get the more accurate results by using various enhancement and segmentation techniques.

Key words: MATLAB, FCM, ANN, CT

Corresponding Author: Ms. Sreesha Govind

\section{INTRODUCTION}

In the beginning of the 20th century, lung cancer was an uncommon disease, but now it's a worldwide dilemma, which is the more recurrent cancer in the world [1]. The lung cancer remains a leading cause of increase in cancer related deaths. The most critical aspect related to the occurrence of cancer is the use of tobacco. Persistent of cough, shortness in breathing, pain in the chest, and bronchitis were some of the symptoms exist when the cancer is in advanced stage. It was found that if the disease was diagnosed earlier, about one-third of cancers become preventable, another one-third become potentially curable.Primary lung cancer is the cancer that originates in the lung. Lung Cancer and Non Small Cell Lung Cancer are the categories of lung cancer. Small Cell Lung Cancer usually starts near the center of the chest in the bronchi . These cancer cell are small in size often spreads very early and is caused by smoking and it will not affect someone who has never smoked. Non small cell lung cancer behaves in a similar way to small cell. Non Small Cell Lung Cancer is diagnosed about 80 to 90 percent of total lung cancer. 
DOI: https://dx.doi.org/10.26808/rs.ca.i8v1.11 International Journal of Computer Application (2250-1797)

Issue 8 Volume 1, January- February 2018

Effective implementation and knowledge from successful research can only pave the way to control the lung cancer [2]. In medical imaging, detection of lung lesion is one of the most challenging tasks [3]. By neighboring anatomical structures, the pulmonary nodule occurs in lung as a spherically shaped mass will become dull to be able to get recognized. The nodule sizes, density and contrast modification creates complexity for spotting lung nodules in CT images. The diameter of a nodule can be in between a few millimeters up to some centimeters. Cancer detection system has been found as a new step in early diagnosis of lung cancer.

Examination of lung tumour is performed with various imaging modalities such as Radiography, Computed Tomography (CT) and Positron Emission Tomography(PET). Among them CT is most frequently used imaging modality due to low cost, quality and robustness. Due to non-pathological structures the radiologist finds difficult and time-consuming to distinguish some nodules in CT [4]. To overcome this, radiologists choose alternate choice to confirm their analysis. Computer- Aided Diagnosis (CAD) systems have been developed toward the intention in medical imaging modalities, to enhance the performance of the diagnosis and improve methodical decisions in clinical practice. It also stresses that methods based on supervised classification generally outperform well compared with other standard methods [5]. According to researchers, support vector machine (SVM) is an imperative task to solve many classical problems.

Our study aims at examining the performance of supervised classifier for the detection of lesions even at small diameter. These classifiers are developed to improve the classification rate and reduce False Positives (FPs).The proposed system consists of the following stages: (i) Extraction of lung region (ii) Segmentation of extracted lung (iii) Detection of nodule (iv) Feature extraction (v) Classification with Artificial Neural Network. Some nodules are rather denser than the neighboring lung tissues. Hence, the visibility of nodule on the CT image is reduced. As a result of contrast variation to the background, the nodule can be found anywhere in the lung field. Lung cancer survival rate is $15 \%$ in five years since a tumor caused a lung cancer by multiplying and developing abnormal cells.

\section{RELATED WORK}

A number of literatures are available to state the problem which arises in automatic detection of pulmonary nodule. Some of the works are presented below:

The methodology developed by Jacobs et al. [6] aims to detect sub solid nodule automatically by means of 128 features based on intensity, shape, texture and context features. This methodology uses Gentle Boost (GB) classifier, Support vector machine (SVM) with radial basis as kernel function, linear discriminant classifier, k-nearest neighbour classifier and nearest mean classifier. The GB- 10 presents better results with $80 \%$ sensitivity and 1 false positive/ scan. The work by Lee et al. [7] developed a two-step feature selection and Random Subspace Method (RSM) for the diagnosis of pulmonary nodules. To validate the performance a set of 216 features for 128 pulmonary nodules were used. The proposed methodology achieves a maximum Az of 8.89 for two-step feature selection and 8.66 for RSM. The architecture of CAD system developed by Silva et al. [8] presents a lung nodule detection system by means of segmentation and classification with Lib SVM. The proposed methodology achieved $84.84 \%$ sensitivity, $96.15 \%$ specificity and $95.21 \%$ accuracy for 33 exams. Liu et al. [9] develop a new CAD system using proprietary databases. In their work, automatic segmentation of CT lung image was performed using thresholding and region growing algorithm. The lung nodule was detected using semi-supervised method called ADE-CoForest with a sensitivity of $85.6 \%$ and false positive of $13.4 \%$. The proposed CAD shows better superiority when compared with SVM.

Choi et al. [10] developed a automatic CAD to improve the detection accuracy for solitary pulmonary nodules. Volumetric segmentation of lung was performed using optimal thresholding 
DOI: https://dx.doi.org/10.26808/rs.ca.i8v1.11 International Journal of Computer Application (2250-1797)

Issue 8 Volume 1, January- February 2018

and neighborhood structures are eliminated using 3D-connected component analysis. For nodule detection a set of techniques such as multi-scale dot enhancement filtering and angular histograms are used to describe the detected features. Finally, a classification approach of SVM was used to obtain a sensitivity of $97.5 \%$ and $6.76 \%$ per scan of false positives.

To evaluate and compare the scheme of other published CAD schemes Silva et al. [11] developed quality threshold algorithm and region growing for image segmentation. The maximum classification sensitivity is $85.91 \%$, specificity $97.7 \%$, accuracy $97.55 \%$ with FP of 1.82 for SVM classifier. Tong et al. [12] has used a three step process to detect lung nodules. Firstly, to segment the lung region an adaptive threshold algorithm was used.Then, to remove lung vessel ,active contour model (ACM) was used and finally a Hessian matrix (selective shape filter) was used to detect the suspicious nodules. This method was able to produce an overall detection rate of $85 \%$.

Marten et al. [13] identified and compared some features as margin, nodule size, position matrix characteristics, vascular and pleural attachments with gold standard. Some author uses manually segmented lesion as gold standard and some others uses specialist references as gold standard. Azimifar et al. [14] used active contour modeling for image segmentation to produce a possible detection rate of $89 \%$.

Hua et al. [15] proposes a deep learning approach in comparison with conventional CAD. The author finds some limitation in CAD. According to their view, the proposed learning approach has some intrinsic advantage in the case of performance evolution. Similarly in the place of NNs, Deep Belief Network (DBN) classifier was designed. Various techniques proposed in CAD were compared against the deep learning approach. The system shows an efficient output when compared with CAD techniques. The authors suggest that this method can be replaced in the place of CAD. Yet, the sensitivity and specificity of this method was $73.4 \%$ and $82.2 \%$ respectively. The proposed DBN was compared with convolutional Neural Network (CNN), Scale Invariant Feature Transform (SIFT) and fractal method.

Firmino et al. [16] proposed a new CAD for the detection of pulmonary nodule. The images are taken from LIDC-IDRI databases and 420 cases are chosen. The nodule type such as solid, pleural, vascular and non solid are selected. The author uses watershed segmentation in order to detect the nodule from neighbouring structures. Histogram of oriented Gradients (HOG) approach was used for extracting the features from the ROI. Various classifiers such as SVM and rule based method was used for reducing FPs. The system achieves an accuracy of 97\%, 94.4\% sensitivity with $7.04 \mathrm{FP} /$ case. Researchers interested in more specific topic about CT images can refer to publications by Irmark et al. for image enhancement algorithm [17], brain tumor [18], and Ertas et al. [19-21] for corticalbone simulation.

Summrina Kanwal Wajid et al. [2012] stated that the Local Energy based Shape Histogram (LESH) feature extraction technique the segmentation of the lung region due to the limitation regarding on the similarities of the intensity in the X-ray image. Zare et al. [2011] declared that the approaches of content- based image retrieval (CBIR) using low level features such as shape and texture are investigated in order to create a framework that classify medical X-ray image automatically. Gray level Co- occurrence Matrix, Canny Edge Operator, Local Binary Pattern and pixel level information of the images act as image based feature representations. The performance of image classification offered by combining the promising features stated above is investigated. Experimental results using 116 different classes of 11,000 X-ray images showed $90.7 \%$ classification accuracy.

\section{MATERIALS AND METHODS}

The developed CAD consists of various stages of methodologies such as (1) CT lung image acquisition, (2) detection of lung lesions, (3) features extraction using texture analysis and (4) 
classification of nodule candidates using supervised algorithm. Fig. 1 displays the flowchart of proposed CAD.

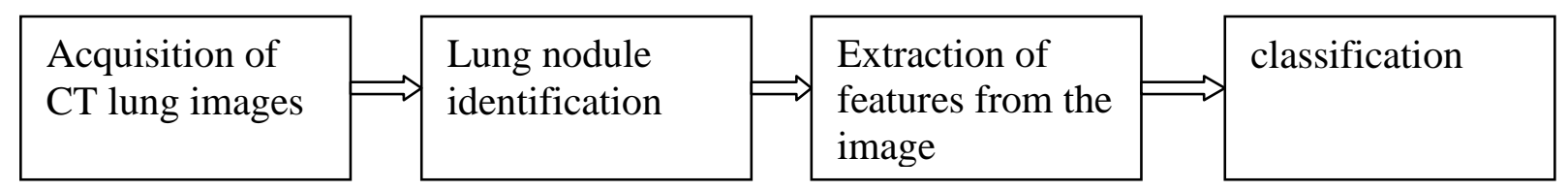

Fig.1: Flow chart of proposed CAD system.

\subsection{CT lung acquisition}

The Lung images are acquired from the Lung Imaging Database Consortium-Image Database Resource Initiative (LIDC-IDRI) [22]. All the images are in DICOM format with the image size of 512 _ 512 pixels. In LIDC-IDRI the thickness of slice varies from 1.25 to $2.5 \mathrm{~mm}$ range with the pixel size from $0.48 \mathrm{~mm}$ to $0.72 \mathrm{~mm}$ respectively [23]. Fig. 2 shows a sample of input images.

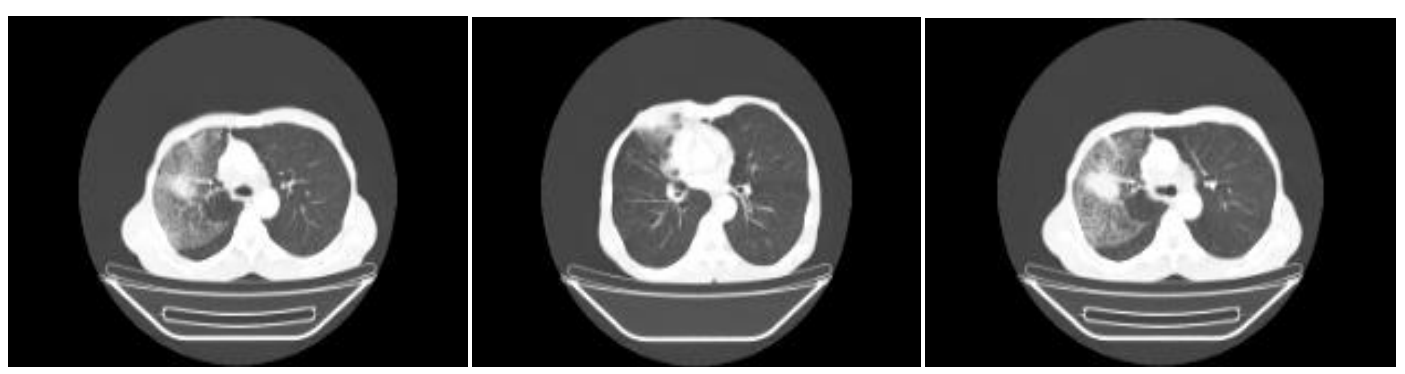

Fig 2.samples of input images

\subsection{Detection of lung lesion}

Detection of lung lesion alone of a CT image is a crucial step. The reason behind is that the intensity of bronchi and blood vessel around the tumour is similar. It is essential to segment the lung parenchyma from the surrounding structures such as heart, diaphragm, bones, skin, fat and tissue. From the segmented parenchyma, it becomes easier to segment the tumour candidates.

The steps involved in detection of lesion as follows.

\subsubsection{Segmentation of lung parenchyma}

Segmentation of Parenchyma from CT images only paves way to identify the tumour easier to diagnose lung disease. The main objective of this stage is to eliminate the mediastinum, thoracic wall that do not contribute in the reconstruction the parenchyma. The proposed work uses Contour Model (CM) for lung segmentation. Based on its operation, this model can be classified in two approaches. The edge based CM produces small variation in segmentationdue to image gradients. If weak boundary exists, leakage problem arises too [25]. Presence of noise in the boundary cannot be tackled in edge-based approach. Region based CM does not produce boundary leakage problem and hinders the effect of problem proposed methodology uses region based CM for segmentation.

The proposed one is an active contour model which is based on snake active contour model [27]. Before beginning the segmentation process, the input lung image is histogram equalized in order to improve the lower contrast region into higher contrast region (i.e. contrast enhancement). Reduction of noise image and detail function can be achieved by using Gaussian function. The technique applied was called as Gaussian blur (smooth). After enhancing the contrast, two approaches such as Gaussian blur and edge detector functions are performed to improve contouring. 
The proposed work uses a specially designed algorithm, which is designed for reducing noise and, for improvising the system performance.. These functions are used to allow sharper reconstructed image. In this stage, Fuzzy C-Mean (FCM) clustering is used for separating the internal structures from lung.

The algorithm for standard fuzzy c means clustering is as follows in Algorithm1.

\section{Algorithm1:}

1. Initialize $U=\left[u_{i j}\right]$ matrix, $U^{(0)}$

2. At k-step: calculate the centers vectors $C^{(k)}=\left[c_{j}\right]$ with $U^{(k)}$

$$
c_{j}=\frac{\sum_{i=1}^{N} u_{i j}^{m} \cdot x_{i}}{\sum_{i=1}^{N} u_{i}^{m}}
$$

3. Update $U^{(k)}, U^{(k+1)}$

$$
u_{i j}=\frac{1}{\sum_{k=1}^{c}\left(\frac{\left\|x_{i}-c_{j}\right\|}{\left\|x_{i}-c_{k}\right\|}\right)^{\frac{2}{m-1}}}
$$

4. If $\left\|U^{(k+1)}-U^{(k)}\right\|<$ then STOP; otherwise return to step 2

Image segmentation is the procedure in which the original image is partitioned into homogeneous regions and plays an important role in medical image processing. As the imaging mechanism and the tissues of medical images are different, medical images are easily affected by noise, field migration effect, and tissue movement. Fuzzy c-means (FCM) is a segmentation method applied to images from the medical field.The performance of this method to obtain an optimal solution depends on the initial positions of the centers of the clusters, the measure of membership degree for each data point, and so on. In the standard FCM, the centers are initialized randomly and the measure of membership only uses the gray feature. This leads to be quite time-consuming and be sensitive to noise. In order to overcome the defect that the FCM is easy to fall into local optimal solution, some researchers combine the FCM and other mathematical approaches .Nevertheless, due to their complexities and poor portabilities, effective medical image segmentation methods are yet to be seen.

\section{PROPOSED SYSTEM}

Inorder to reduce noise affected to the medical images before applying it into FCM it is intended to introduce a new methodology called as Spacial-noise removal. 


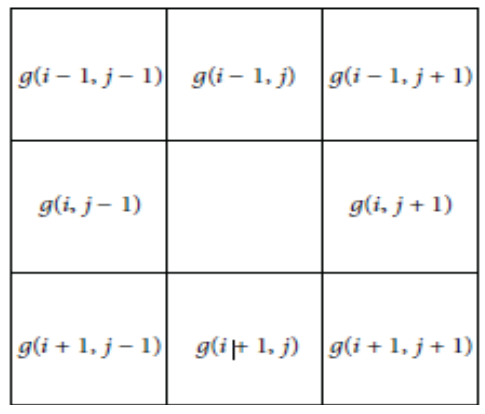

Fig 3. Neighbour regions of $g(i, j)$.

Step 1: A random matrix consisting of pixels in the range 0-255 is made of any dimension.

Step 2: A 3x3 matrix from the random matrix is chosen.

Step 3: Values in the $3 \times 3$ matrix is sorted. The end result after sorting will be as minimum value pixel occupy first row, middle value of 9 elements in the selected $3 \times 3$ matrix from random matrix will occupy the middle position of the matrix, maximum value at the last position.

Step 4: For a pixel $x(i, j)$ to be a normal pixel, if it lies between the minimum and maximum value of 3x3 matrix and here maximum value must be less than 255 and minimum value must be greater than 0 .

Step 5:if $x(i, j)$ is a noise pixel,(ie, if it fails the above condition), it will be replaced by the middle value of the 9 elements. Then the middle value lies between the maximum and minimum value of the $3 \times 3$ matrix and also the middle value lies between $0 \& 255$

Step 6:if the above condition is not true for middle value, ie, middle value is greater than both maximum and minimum value of $3 \times 3$ window or its equal to 0 or 255 , then the value will be changed as the value of the left neighbourhood pixel.

step 7: The process is iterated in the matrix to remove as much possible noise.

\subsubsection{Nodule detection}

The extracted ROI consists of structures or nodules. Rule based methods are used for separating the nodule/non nodular structures. Solid nodules are dense and spherical. The roundness rule and high intensity data points are utilized for segmenting solid nodule. Roundness of the object nearer to 1 are considered as solid nodule. Other structure such as blood vessel is excluded using morphological operation. Part solid nodules are less dense and they can be extracted from the medium intensity data points. The intensity of such clusters is increased and segmentation. The region other than nodular part can be excluded using morphological operation. The intensity of non solid nodules are very much lesser than solid nodules. Hence the low intensity cluster data points are detected whereas non structural elements are discarded using morphological operation. But a problem arises in this process is that; lesion that is connected along the chest wall cannot be extracted in the segmented stage. Even though the weak edges are retrieved by the region based contour method the juxtapleural cannot be rescued. To extract the excluded part, reconstruction of lung border was employed. 
DOI: https://dx.doi.org/10.26808/rs.ca.i8v1.11 International Journal of Computer Application (2250-1797)

Issue 8 Volume 1, January- February 2018

\subsubsection{Border correction}

The reconstruction of the lung border is main steps that aim to recover lung nodules that are attached to the thoracic wall. The Adaptive border marching, greedy snake algorithm , ray casting, vector quantization, and chain codes etc can be used to recover the juxta pleural nodules. The present work employs a technique called rolling ball, which uses morphological closing operations with a spherical structuring elemental of $10 \mathrm{~mm}$ along the contour of the lung, causing the reconstruction of the concavities where this element cannot enter. The rolling ball approach was most successful for recovering the juxtapleural nodule.

\subsubsection{Separation of vascular nodule}

Vascularized nodules are located in the central lung connected with the blood vessels. Using region based ACM lung can be segmented .FCM is used for extracting the ROI. Spherical objects can be identified using roundness rule. Separations of larger and smaller vascular nodules are based on morphological operations.

\subsection{Features extraction using texture analysis}

Feature of an image was computed to produce one or more quantity, and each quantity specifies the property of a particular image. Features can be analyzed using various techniques. They are spatial based; transform based; shape based; intensity based; edge based, etc. Two set of intensity based techniques such as first order statistics and second order statistics have to be used in this work.

\subsubsection{First order statistics (FOS)}

First order feature can also be called as histogram based features. These features are calculated based on the individual pixel and not by neighboring pixels. Mean, variance, entropy, energy and skewness are the five important FOS studied in this work.

\subsubsection{Second order statistics (SOS)}

The GLCM is a well-established second order statistical technique which is used for extracting texture feature from ROI. Extraction of various images such as brain, liver, lung, etc. often uses GLCM texture analysis method, due to the capturing ability of gray level in the image. A GLCM matrix consists of rows and columns with equal number of dissimilar gray levels of the particular image. Featuring of tumor based on its shape, size, shape or intensity was hard, because the intensity for small tissues overlap in CT images. The shape and size of each tumor is not uniform for all slices. Therefore, to measure the statistical properties of pixel information, regularity, smoothness of nodules texture features can only be used.

The extracted features of lung lesions are based on the analysis of gray level co-occurrence matrix (GLCM). The size of the GLCM can be calculated from the gray level of an image. Pixel relationship between different direction $(0 \mathrm{~L}, 45 \mathrm{~L}, 90 \mathrm{~L}, 135 \mathrm{~L})$ and distance gives the Offsets of GLCM. It was noted that single offsets suffers due to sensitive of texture measurements. Hence for every input image multiple GLCM offset has been found. These features help us to identify the lung nodule from the CT images.

The GLCM features such as maximum probability, difference, entropy, autocorrelation, contrast, correlation, cluster prominence, cluster shade, dissimilarity, homogeneity, sum of squares, sum average, information measure of correlation2, and inverse difference normalized. sum variance, sum entropy, difference variance, information measure of correlation1, has been studied.

\subsection{Classification of nodule candidates-SVM classifier}

Classification is the important and the last stage of the CAD sys-tem. The classifier 
distinguishes normal tumor from abnormal tumor.

TP (True Positive) represents presence of disease identified by both CAD and physicians, FN (False Negative) shows the absence of disease by CAD but presence of disease by physicians, FP (False Positive) shows the presence of disease by CAD but absence of disease by physicians and TN (True Negative) represents absence of disease identified by both CAD and physicians. Accuracy is obtained by $(\mathrm{TP}+\mathrm{TN}) /(\mathrm{TP}+\mathrm{TN}+\mathrm{FP}+\mathrm{FN})$. Sensitivity is obtained by TP/(TP + FN), Specificity is given by TN/ $(\mathrm{TN}+\mathrm{FP})$.

\section{RESULTS}

In this section, the results achieved from different modules of the project are discussed.Result before and after the application of new noise removal algorithm(proposed algorithm)has been shown in images below in Fig 4.

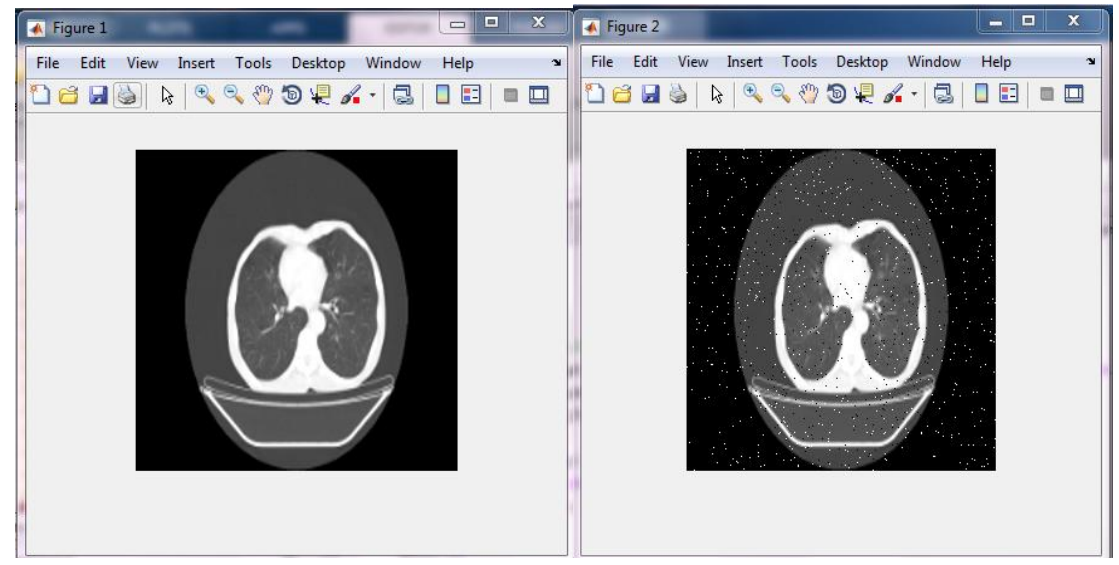

(a)

(b)

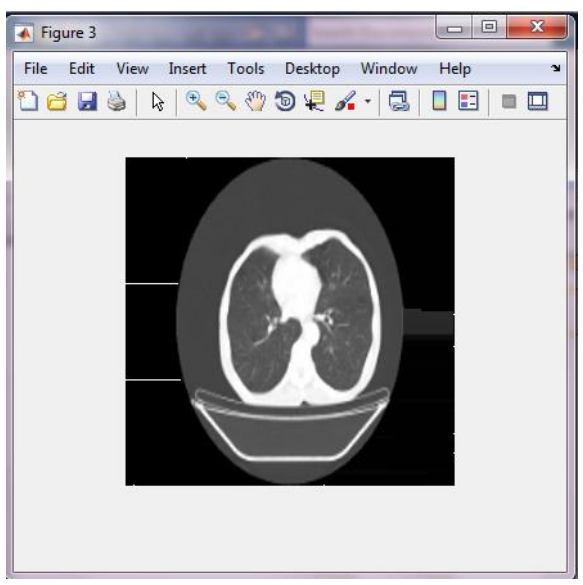

(c)

Fig 4. (a) Original image (b) Noise added to image (c) Noise removed from image

A comparison Table 1 showing the results before and after noise removal also included to prove the efficiency.After applying this algorithm, the PSNR value obtained by comparing the initial and noise removed images has been become 21.73 and before the removal of noise the PSNR value between the original image and noisy image was obtained as 40.097. This shows the improvement after applying the algorithm. 
Table 1:PSNR comparison

\begin{tabular}{|c|l|c|}
\hline $\begin{array}{c}\text { Image } \\
\text { number }\end{array}$ & \multicolumn{1}{|c|}{ Image } & PSNR value \\
\hline 1 & $\begin{array}{l}\text { Original Lung CT image with noisy image(salt } \\
\& \text { pepper) }\end{array}$ & 21.7373 \\
\hline & Original lung CT image with noise removed image & 40.0974 \\
\hline 2 & $\begin{array}{l}\text { Original Lung CT image with noisy image(salt } \\
\& \text { pepper) }\end{array}$ & 21.1955 \\
\hline 3 & $\begin{array}{l}\text { Original lung CT image with noise removed image } \\
\text { \&pepper) }\end{array}$ & 38.1053 \\
\hline & Original lung CT image with noise removed image & 22.5168 \\
\hline
\end{tabular}

The application of snake based active contour model will produce results as follows in Fig 5.

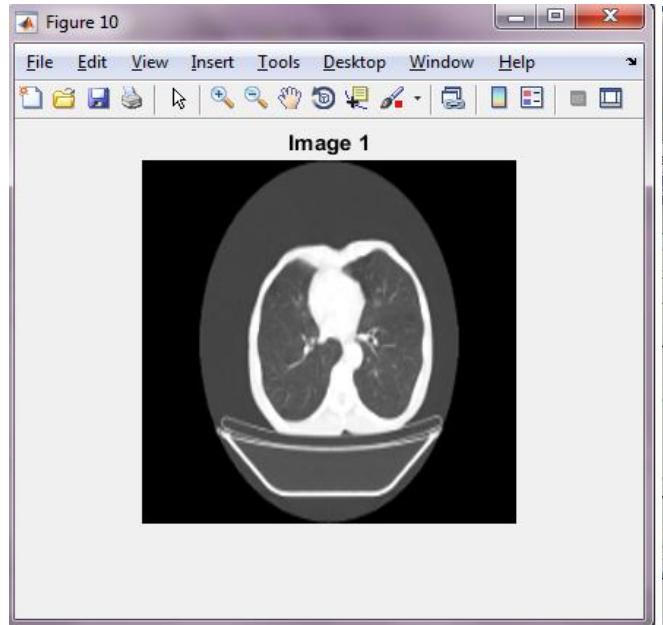

(a)

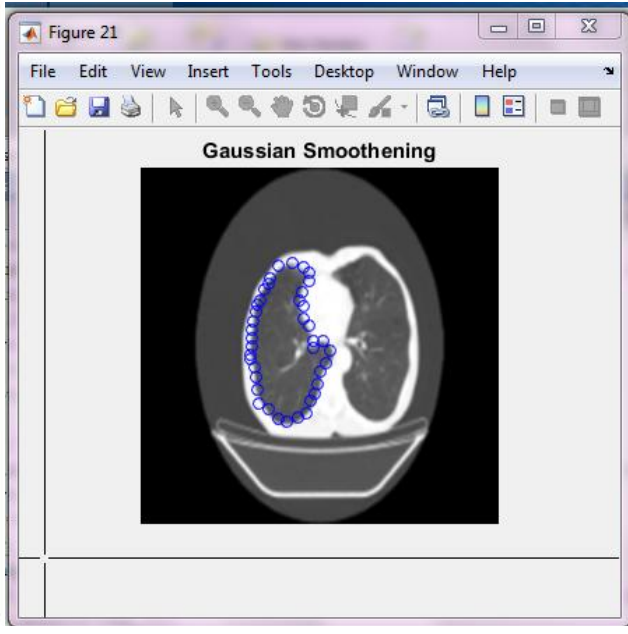

(b)

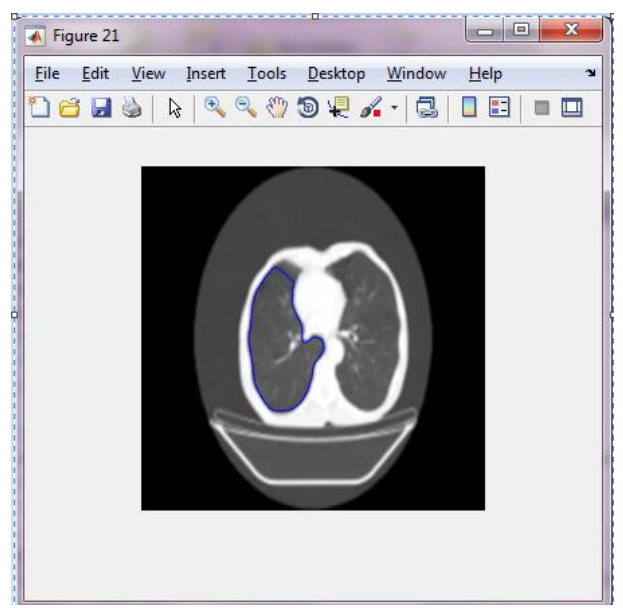

(c)

Fig 5: Snake based active contour model

\section{CONCLUSION}

The paper presents support vector machine classifier based pulmonary nodule detection methodology. The proposed method is expected to show a reduced FPR, improved sensitivity with reduced computation time. An early diagnosis can result in a considerable increase in the survival 
chance of patients. The proposed methodology suggests that the developed CAD system has great potential and promise in the automatic diagnosis of tumors of lung.

\section{REFERENCES}

[1] American Cancer Society, Cancer Facts \& Figures, 2015.

[2] M. Krishnan Nair, Cherian Varghes, R. Swaminathan, Cancer: Current scenario, intervention strategies and projections for 2015.

[3] Nitin S. Lingayat, Manoj R. Tarambale, A computer based feature extraction of lung nodule in chest X-ray image, Int. J. Biosci., Biochem. Bioinf. 3 (6) (2013) 624-629, http://dx.doi.org/10.7763/IJBBB.2013.V3.289.

[4] G. Gargano, R. Bellotti, et al., A CAD system for nodule detection in low-dose lung CTs based on region growing and a new active contour model, Med. Phys. 34 (12) (2007) 49014910, http://dx.doi.org/10.1118/1.2804720.

[5] Carole Lartizien, Simon Marache-Francisco, Rémy Prost, Automatic detection of lung and liver lesions in 3-D positron emission tomography images: a pilot study, IEEE Trans. Nucl. Sci. 59 (1) (2012) 102-112.

[6] Colin Jacobs, Eva M. van Rikxoort, Thorsten Twellmann, Ernst Th. Scholten, Pim A. de Jong, Automatic detection of subsolid pulmonary nodules in thoracic computed tomography images, Med. Image Anal. 18 (2014) 374-384.

[7] Michael C. Lee, Lilla Boroczky, Kivilcim Sungur-Stasik, Aaron D. Cann, Alain C. Borczuk, Computer-aided diagnosis of pulmonary nodules using a two-step approach for feature selection and classifier ensemble construction, Artif. Intell. Med. 50 (2010) 43-53.

[8] Aristófanes Correa Silva, João Rodrigo Ferreira da Silva Sousa, Anselmo Cardoso de Paivaa, Rodolfo Acatauassú Nunes, Methodology for automatic detection of lung nodules in computerized tomography images, Comp. Meth. Prog. Biomed. 98 (2010) 1-14.

[9] Yang Liu, Zhian Xing, Chao Deng, Ping Li, Maozu Guo, Automatically detecting lung nodules based on shape descriptor and semi supervised learning, in: International Conference on Computer Application and System Modeling (ICCASM 2010), pp. 647-650.

[10] Tae-Sun Choi, Automated pulmonary nodule detection based on three-dimensional shapebased feature descriptor, Comput. Methods Programs Biomed. 113 (2014) 37-54.

[11] Aristófanes Corrêa Silva, Automatic detection of solitary lung nodules using quality threshold clustering, genetic algorithm and diversity index, Artif. Intell. Med. 60 (2014) 165177.

[12] Jia Tong, Wei Yin, Wu Cheng Dong. A Lung cancer lesions detection scheme based on CT image, in: International Conference on Signal Processing Systems, 2010, pp. 557-560.

[13] K. Martena, C. Engelke, T. Seyfarth, A. Grillho€sl, S. Obenauer, E.J. Rummeny, Computeraided detection of pulmonary nodules: influence of nodule characteristics on detection performance, Clin. Radiol. 60 (2005) 196-206.

[14] Zohreh Azimifar, Mohsen Keshani, Farshad Tajeripour, Reza Boostani, Lung nodule segmentation and recognition using SVM classifier and active contour modeling: a complete intelligent system, Comput. Biol. Med. 43 (2013) 287- 300.

[15] Kai-Lung Hua, Che-Hao Hsu, Shintami Chusnul Hidayati, Wen-Huang Cheng, Yu-Jen Chen, Computer-aided classification of lung nodules on computed tomography images via deep learning technique, Onco Targets Ther. 8 (2015) 2015-2022.

[16] Macedo Firmino, Giovani Angelo, Higor Morais, Marcel R. Dantas, Ricardo Valentim, Computer-aided detection (CADe) and diagnosis (CADx) system for lung cancer with likelihood 
of malignancy, BioMed. Eng. OnLine 15 (2016) 2.

[17] E. Irmak, A.H. Ertas, A review of robust image enhancement algorithms and their applications, Smart Energy Grid Eng. (SEGE) (2016) 371-375.

[18] E. Irmak, Ergun Erçelebi, Ahmet Hanifi Ertas, Brain tumor detection using monomodal intensity based medical image registration and MATLAB, Turk. J. Elec. Eng. Comp. Sci. 24 (2016) 2730-2746.

[19] A.H. Ertas, K. Winwood, P. Zioupos, J.R. Cotton, Simulation of creep in non-homogenous samples of human cortical bone, Comp. Meth. Biomech. Biomed. Eng. 15 (10) (2012) 11211128.

[20] A.H. Ertas, B.J. Sindelar, J.R. Cotton, Creep simulation of a micro-CT based finite element model of porcine cancellous bone, in: ASME 2011 Summer Bioengineering Conference, pp. 285-286.

[21] A.H. Ertas, Creep simulation and comparison of micro-CT-based FE model of porcine and human cancellous bone, Comp. Meth. Biomech. Biomed. Eng. 16 (sup 1) 340-341.

[22] Samuel G. Armato, Lubomir Hadjiiski, Georgia D. Tourassi, Karen Drukker, Maryellen L. Giger, Feng Li, George Redmond, Keyvan Farahani, Justin S. Kirby, Laurence P. Clarke, Guest Editorial: LUNGx challenge for computerized lung nodule classification: reflections and lessons learned, J. Med. Imag. 2 (2) (2015).

[23] S.L.A. Lee, A.Z. Kouzani, E.J. Hu, Automated Identification of Lung Nodules, MMSP, 2008, pp. 497-502.

[24] Sarah Taghavi Namin, Hamid Abrishami Moghaddam, Reza Jafari et al., Automated detection and classification of pulmonary nodules in 3D thoracic CT images, in: 2010 IEEE, pp. 3374-3379.

[25] Wonjun Kim, Changick Kim, Active contours driven by the salient edge energy model, IEEE Trans. Image Process. 22 (4) (2013) 1667-1673, http://dx.doi.org/ 10.1109/TIP.2012.2231689.

[26] E.E. Nithila, S.S. Kumar, Segmentation of lung nodule in CT data using active contour model and Fuzzy C-mean clustering, Alex. Eng. J. (2016), http://dx.doi. org/10.1016/j.aej.2016.06.002

[27] "snakes :active contour model", Michael kass,Andrew witkin and demitri terzopoulos 